\title{
Cerebral Blood Flow in Sneddon Syndrome
}

\author{
Christian Menzel, Uwe Reinhold, Frank Grünwald, Alexander von Smekal, Manfred Uerlich, Olaf Rieker, \\ Andreas L. Hotze and Hans J. Biersack
}

Departments of Nuclear Medicine and Dermatology, University of Bonm, Bonm, Germany

\begin{abstract}
Sneddon syndrome is defined as a clinical entity consisting of livedo racemosa generalisata (LRG) and cerebrovascular lesions, which often lead to physical and mental handicaps. Four patients with LRG and the suspected diagnosis of Sneddon syndrome had HMPAO-SPECT studies. The patients underwent $C T$ and/or MR brain imaging and three patients had Duplex sonography of the cerebral arteries (TCD). Brain SPECT was abnormal in all patients, whereas CT/MRI revealed a cerebral lesion in only one patient and all TCD studies were normal. HMPAO-SPECT is valuable in detecting disturbed regional $\mathrm{C \theta}$ rebral blood flow before irreversible ischemic insults occur, thus allowing the diagnosis of Sneddon syndrome at an early stage.

Key Words: technetium-99m-HMPAO; SPECT; Sneddon syndrome; rCBF
\end{abstract}

J Nucl Med 1994; 35:461-464

A complex of symptoms consisting of livedo racemosa generalisata (LRG) and cerebrovascular lesions was first described by J. B. Sneddon in 1965 (1). The etiology of this syndrome still remains unclear. Several authors, however, found an association with vascular risk factors. A correlation with high levels of anticardiolipin antibodies was described recently indicating that localized thrombus and autoimmuno-induced obliteration of normal intimal proliferation may play a role in the pathogenesis of this disease $(2,3)$.

LRG is characterized as an occlusive, inflammatory disorder of smaller arteries involving subcutaneous and deep dermis leading to "flash-like" and arborizing cutaneous figures (4). The LRG shows an autosomal dominant inheritance with a marked prevalence in younger females (5). It is also associated with peripheral ischemic features such as Raynaud phenomenon and acrocyanosis.

Cutaneous symptoms usually precede the neurologic manifestations of the disease such as atrophy, stroke, TIA, PRIND or epilepsy, occasionally by many years. The cerebrovascular disease, leading to multiple cerebral infarctions, often results in mental impairment or dementia. This demonstrates the possible severity of the disease.

Received Aug. 31, 1993; revision accepted Nov. 29, 1993.

For correspondence or reprints contact: Christian Menzel, MD, Dept. of Nuclear Medicine, University of Bonn, Sigmund Freud Str. 25, 5300 Bonn 1 Germany.
Due to an unexplained etiology, the treatment of Sneddon syndrome is currently unsatisfactory. The improvement of blood rheology seems to be the major pharmacological approach to the treatment of this syndrome in order to avoid or at least delay the onset of nonreversible cerebral damage.

Since the histological diagnosis of LRG in this syndrome is often uncertain and morphologic cerebral alterations seen with CT/MRI represent mainly irreversible lesions, the evaluation of cerebral blood flow may be of diagnostic relevance. HMPAO is recognized as a tracer for cerebral blood flow measurement with an especially high reliability in detecting alterations caused by cerebrovascular disease $(6,7)$.

We investigated four patients with LRG suspected to suffer from Sneddon syndrome. HMPAO-brain SPECT imaging was performed with a high-resolution SPECT system. All patients had CT and/or MRI, transcranial duplex sonography and a full neurological examination.

\section{MATERIALS AND METHODS}

We investigated cerebral blood flow in four female patients (mean age $38 \mathrm{yr}$ ) with known LRG using ${ }^{99 \mathrm{~m}} \mathrm{Tc}$-HMPAO-SPECT.

\section{Dermatological Examination}

Diagnosis of LRG was established by a clinical dermatologist on basis of typical cutaneous symptoms described elsewhere (13). Histological evaluation of skin lesions using deep cutaneous lancet biopsy under local anesthesia was performed in all patients. Other systemic diseases and factors associated with LRG were excluded by intensive clinical and laboratory investigations.

\section{Neurological Examination}

The clinical neurological status was assessed by an experienced neurologist. The evaluation included testing sensory and motor defects of central and peripheral nerves as well as a clinical examination of mental functions.

\section{Radiological Examination}

Radiological evaluation was obtained via native CT brain scans (Somatom, Siemens) and/or MRI-studies (Gyroscan 1.5 T, Phillips) of the brain. The MRI studies included T1-weighted sagittal scouts, proton- and $\mathrm{T} 2$-weighted transverse scans and eventually coronal inversion recovery scans. In Patient 2 , only contrast media were used in CT and MRI. The MRI study of this patient consequently included $\mathrm{T} 1$-weighted transversal and coronal slices before and after the application of Gd-DTPA. 
TABLE 1

Clinical and Laboratory Data

\begin{tabular}{|c|c|c|c|c|c|c|c|c|c|}
\hline & Sex & Age & H & $A C L$ & ANA & CRP & RF & TPHA & Risk factors for vascular sclerosis \\
\hline C1 & $f$ & 49 & + & - & - & + & - & - & $\begin{array}{l}\text { Hypercholesterinemia, } \\
\text { hypertension, nicotine abuse }\end{array}$ \\
\hline C2 & $f$ & 35 & + & - & - & + & - & - & None \\
\hline C3 & $f$ & 26 & - & - & - & - & - & - & $\begin{array}{l}\text { Hypercholesterinemia, nicotine } \\
\text { abuse }\end{array}$ \\
\hline $\mathrm{C4}$ & $f$ & 42 & - & + & - & - & - & - & $\begin{array}{l}\text { Increased platelet aggregation in } \\
\text { response to ADP and } \\
\text { ristocetin, nicotine abuse }\end{array}$ \\
\hline
\end{tabular}

$H=$ histologically proven LRG; $A C L=$ anti-cardiolipin antibodies; $A N A=$ anti-nuclear antibodies; $C R P=C$-reactive protein; RF = rheumatoid factor, TPHA = treponema pallidum hemagglutination test; and ADP = adenosine-di-phosphate.

\section{SPECT Evaluation of Cerebral Perfusion}

Regional cerebral blood flow was measured by HMPAO brain SPECT imaging. The tracer was administered in a dark, quiet room with the patient's eyes open. Acquisition with an annular high-resolution SPECT system and a standard three-field, lowenergy, high-resolution collimator (Ceraspect, DSI) was started 10-30 min after intravenous injection of $740 \mathrm{MBq}(20 \mathrm{mCi})$ of ${ }^{99} \mathrm{~m}$ Tc-HMPAO. Spatial resolutions at $140 \mathrm{keV}$ measured the FWHM of the system at $<8.6 \mathrm{~mm}$ at center of rotation and $6.5-7$ $\mathrm{mm}$ for cortical regions. Sagittal, coronal and transverse slices were reconstructed using a Butterworth filter. The cerebral perfusion patterns were evaluated by two experienced nuclear medicine specialists.

\section{RESULTS}

Diagnosis of LRG was established clinically in all patients. Raynaud phenomenon was evident in $2 / 4$ patients (Patients 3 and 4). Perivascular lymphocyte infiltrations in the deep dermis were detected in 2/4 patients. Anti-cardiolipin-AB were moderately elevated in $1 / 4$ patients (Patient 4). Blood sedimentation rate (BSR) and C-reactive protein (CRP) were slightly elevated in $2 / 4$ patients. Other parameters of autoimmuno-induced inflammatory processes, such as anti-nuclear-antibodies, rheumatoid factor (RF) and TPHA were negative and $3 / 4$ patients had a moderate to severe (Patient 3 ) risk for vascular sclerosis (Table 1 ).

Epilepsy was evident in Patients 1 and 2. Both patients, however, were free of seizures for more than 1 wk before brain SPECT. Patient 3 showed a slight accentuation of the reflexes of the left arm and leg. Patient 4 was neurologically inconspicuous. Furthermore, no additional neurologic defects could be detected in Patients 1-3. In Patient 1, radiological examination (MRI) of the brain was normal. In Patient 2, CT/MRI demonstrated a substance defect in the left parietal region due to vascular occlusion and a process within the tentorium cerebelli. This process appeared hyperdense on native CT scans and a signal decrease was noted on proton- and T2-weighted MRI images in this location. Using contrast media, this process showed a contrast/signal enhancement in its peripheral parts in CT/MRI. Angiography of the cerebral arteries (Seldinger's technique) could not detect any pathological blood vessels. The etiology of this process remains unclear. However, a malignoma or metastasis does not seem likely as the process was described to be avascular. The patient remains under close clinical follow-up.

Patient 3 showed a general cortical atrophy without any additional signs of circumscript cortical substance defects. Patient 4 had no detectable cortical defects using MRI.

Transcranial duplex sonography (TCD) of the cerebral arteries was performed in all patients except Patient 3 and showed no pathological result in any of these patients.

HMPAO-SPECT detected areas of impaired cerebral perfusion in all patients. Patient 1 had a large left parietal defect and a relative hypoperfusion of the frontal cortex. The corresponding MRI was normal (Fig. 1 A-D). Patient 2 showed perfusion defects in the left frontal, temporal and-consistent with CT and MRI findings-left parietal regions. Patient 3 showed distinct defects in the left parieto-occipital and left temporal areas. In all three patients with slight cortical atrophy, no ischemic lesions were noted on CT. Patient 4 had a widely normal distribution throughout the whole cortex revealing only a minimal perfusion defect within the right occipital region (Table 2).

\section{DISCUSSION}

LRG is a dermatological manifestation consisting of superficial venous stasis which often is the first symptom of Sneddon syndrome, usually preceding neurologic manifestations of the disease by years (8). Differentiation of the harmless livedo reticularis is clinically possible by histological demonstration of perivascular lymphocyte infiltrations and endothelial proliferations of smaller and middlesize cutaneous arteries $(8,9)$. Biopsy, however, is difficult because morphologic alterations are minimal and often missed. Clinically and histologically, these alterations manifest as vasculitis but no typical serologic parameters can be found $(4,10)$.

Since 1965, Sneddon syndrome has been defined by the combination of LRG with multiple but mainly ischemic neurologic symptoms. Multiple case reports clearly demonstrate the severity of neurovascular disease often leading to handicaps or multi-infarct dementia (3). Recent studies 

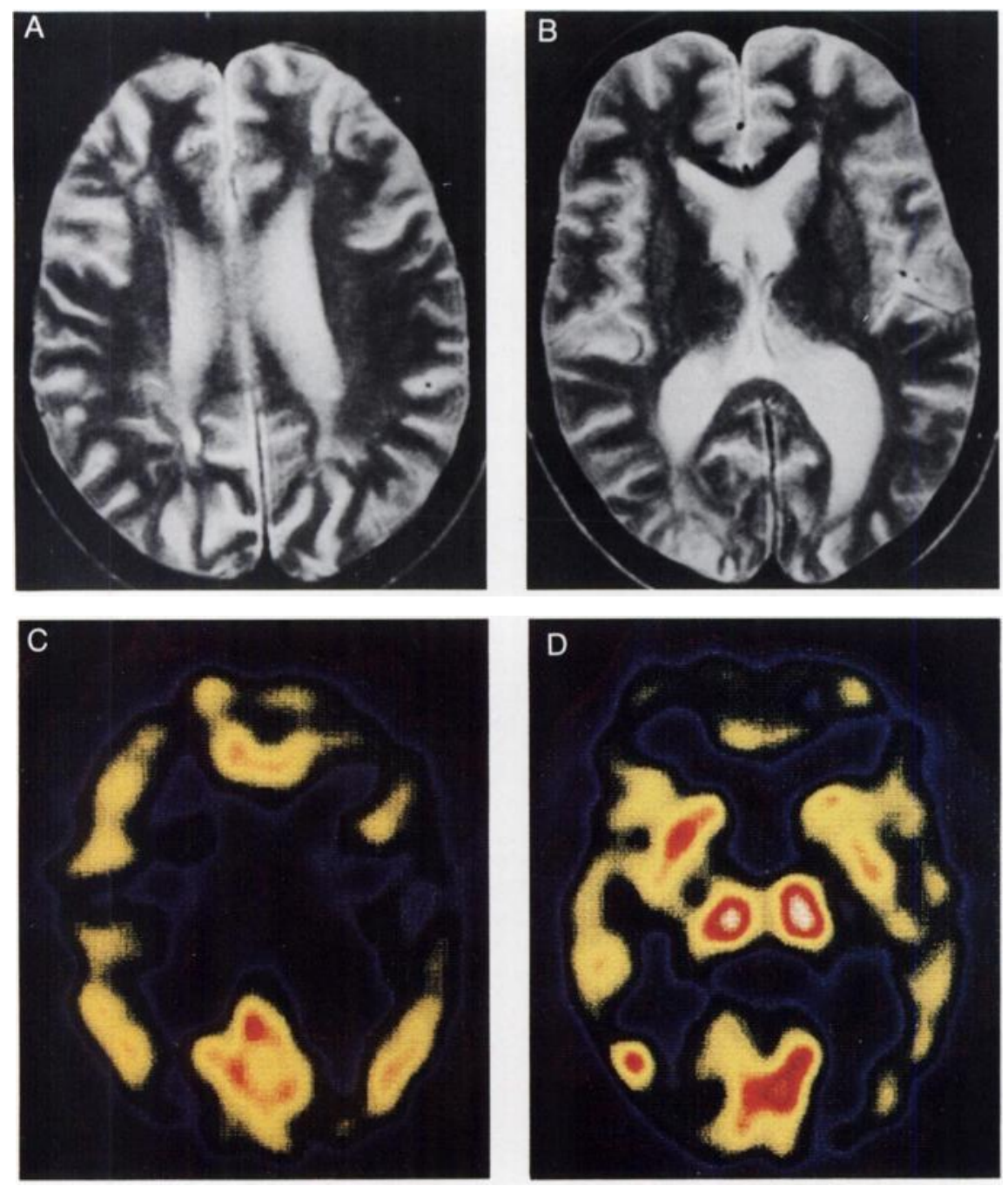

FIGURE 1. Patient 1. T2-weighted transversal MRI slices (A, B) were normal. Corresponding transversal SPECT slices (C, D) showed a large perfusion defect in the left parietal cortex and hypoperfusion of the frontal cortex.

could also demonstrate that the syndrome has a much higher prevalence than expected if all patients suffering from cerebrovascular disease were carefully screened for Sneddon syndrome (5).

Therapeutic approaches to inflammatory diseases such as endangiitis obliterans using corticoids, azathioprine or other immunosuppressive drugs have not yet led to a satisfactory treatment of Sneddon syndrome.

A symptomatic pharmacological approach towards this disease includes the improvement of blood-rheology by means of substances reducing platelet aggregability or improving blood fluidity (11). Radiological procedures (CT/ MRI) allow evaluation of brain morphology, but they do not give any information concerning its functional aspects. Several authors clearly demonstrated the value of HMPAO-SPECT in detecting areas of hypoperfusion caused by cerebrovascular disease $(6,7,12)$.

In this study, Sneddon syndrome was diagnosed in $2 / 4$ patients (Patients 1 and 2) since LRG was histologically proven and neurological symptoms were evident in those

TABLE 2

Location of Pathologic Findings in SPECT, MRI, CT and TCD

\begin{tabular}{|c|c|c|c|c|}
\hline & SPECT & MRI & CT & TCD \\
\hline $\begin{array}{l}\mathrm{C} 1 \\
\mathrm{C} 2 \\
\mathrm{C} 3 \\
\mathrm{C} 4\end{array}$ & $\begin{array}{l}\text { L parietal and bifrontal } \\
\text { L parietal, frontal, temporal } \\
\text { L parieto-occipital, temporal } \\
\text { R occipital }\end{array}$ & $\begin{array}{l}\text { Nomal } \\
\text { L parietal* } \\
\text { Not done } \\
\text { Normal }\end{array}$ & $\begin{array}{l}\text { Not done } \\
\text { L parietal* } \\
\text { Minimal gen. atrophy } \\
\text { Not done }\end{array}$ & $\begin{array}{l}\text { Normal } \\
\text { Normal } \\
\text { Not done } \\
\text { Normal }\end{array}$ \\
\hline
\end{tabular}


patients. In Patient 2, additional morphologic defects could be detected by MRI and CT. Patients 3 and 4 did not show any signs of ischemic cerebral defects using CT or MRI but did have pathological brain SPECT images suggesting cerebrovascular involvement of the disease. Cerebral perfusion reserve by acetazolamid (Diamox ${ }^{\circledR}$ ) or $\mathrm{CO}_{2}$ inhalation could be valuable for even better detection of patients with an elevated cerebrovascular risk because a dilatation of the cerebral arteries could lead to a clearer demarcation of hypoperfused cerebral areas that should already have dilatated vessels due to cerebrovascular auto-regulation. However, neither of these tests was applied in this study. Because cutaneous symptoms precede neurologic manifestations of the syndrome by years, HMPAO-SPECT seems to be a powerful tool to assess cerebral perfusion disturbances at an early stage of the disease. Early symptomatic treatment could then prevent or delay the onset of stroke. Recent studies claimed that headaches or migraines may be the first symptoms, indicating the cerebrovascular progress of Sneddon syndrome (2). On the basis of our study, we suggest that patients with LRG be monitored carefully for the onset of neurologic symptoms and be screened for cerebral perfusion defects using HMPAO-SPECT to exclude or determine the diagnosis of Sneddon syndrome. Patients with an established diagnosis as well as their family members are included since Sneddon syndrome shows an autosomal dominant inheritance.

\section{REFERENCES}

1. Sneddon JB. Cerebrovascular lesions and livedo reticularis. $\mathrm{Br} J$ Dermatol 1965;77:180-185.

2. Martinelli A, Martinelli P, Ippoliti M, Giuliani S, Coccagna G. Sneddon syndrome presenting with hemicranic attacks: a case report. Acta Neurol Scand 1991;83:201-203.

3. Grosse Aldenhövel HB. Das Sneddon-Syndrom-diagnostische, ätiologische und therapeutische Gesichtspunkte. Schweiz Rundschau Med 1990; 23:726-729.

4. Grattan CEH, Burton JL, Boon AP. Sneddon's syndrome with livedo vasculitis and anticardiolipin antibodies. Br J Dermatol 1989;120:441-447.

5. Rebollo M, Val JF, Garijo F, Quintana F, Berciana J. Livedo reticularis and cerebrovascular lesions (Sneddon-syndrome). Brain 1983;106:965-979.

6. Grünwald F, Broich K, Kropp J, Hartmann A, Biersack HJ. SPECT bei reversibler Symptomatik zerebrovasculärer Erkrankungen. Nucl Med 1989; 28:221-225.

7. Podreka I, Suess E, Goldenberg G, et al. Initial experience with ${ }^{99 \mathrm{~m}} \mathrm{Tech}$ netium HMPAO brain SPECT. J Nucl Med 1987;28:1657-1666.

8. Bruyn RPM, van der Veen JPW, Donker AJM, Valk J, Wolters EC. Sneddon's syndrome. Case report and literature review. J Neurol Sci 1987;79: 243-253.

9. Rumple E, Rumple $\mathrm{H}$. Recurrent transient global amnesia in a case with cerebrovascular lesions and livedo reticularis (Sneddon-syndrome). J Neurol 1979;221:127-131.

10. Fan PT, Davis JA, Somer T, Kaplan L, Bluestone R. A clinical approach to systemic vasculitis. Semin Arthitis Rheum 1980;9:248-304.

11. Thomas DJ, Kirby JDT, Britton KE, Galton DJ. Livedo reticularis and neurological lesions. BrJ Dermatol 1982;106:711-712.

12. Grünwald F, Zierz S, Broich K, Schumacher S, Bockisch A, Biersack HJ. HMPAO-SPECT imaging resembling Alzheimer-type dementia in mitochondrial encephalomyopathy with lactat acidosis and stroke like episodes (MELAS). J Nucl Med 1990;31:1740-1742.

13. Copemen FWM. Livedo reticularis: signs in the skin of disturbance of blood viscosity and blood flow. Br J Dermatol 1975;93:519-529. 